\title{
Ubiquitous Nature of Multimode Vibrational Resonances in Positron-Molecule Annihilation
}

\author{
A. C. L. Jones, J. R. Danielson, M. R. Natisin, and C. M. Surko \\ Physics Department, University of California, San Diego, La Jolla California 92093, USA \\ G. F. Gribakin \\ Department of Applied Mathematics and Theoretical Physics, Queen's University, Belfast BT7 1NN, \\ Northern Ireland, United Kingdom \\ (Received 7 December 2011; published 27 February 2012; corrected 22 March 2012)
}

\begin{abstract}
Positron annihilation on many molecules occurs via positron capture into vibrational Feshbach resonances, with annihilation rates often further enhanced by energy transfer to vibrational excitations weakly coupled to the positron continuum. Data presented here uncover another scenario in which the positron couples directly to a quasicontinuum of multimode vibrational states. A model that assumes excitation and escape from a statistically complete ensemble of multimode vibrations is presented that reproduces key features of the data.
\end{abstract}

DOI: 10.1103/PhysRevLett.108.093201

PACS numbers: 34.80.Uv, 34.50.-s, 34.80.Lx

Positron annihilation on atoms and molecules is important in many areas of science and technology including positron emission tomography in medicine, astrophysics, and studies of material properties. For many molecules, the annihilation rates as a function of incident positron energy $\varepsilon$ exhibit vibrational Feshbach resonances (VFRs) (e.g., populated via excitation of infrared-active modes) [1]. These resonances occur at specific values of incident positron energy given by

$$
\varepsilon_{\nu}=\omega_{\nu}-\varepsilon_{b},
$$

where $\omega_{\nu}$ is the vibrational mode energy and $\varepsilon_{b}$ is the positron-molecule binding energy. The observation of these VFRs indicates that the molecule can form a bound state with a positron. The magnitudes of these VFRs are often further enhanced by intramolecular vibrational energy redistribution (IVR). A theory has been developed to explain quantitatively VFR-enhanced annihilation rates in selected small molecules [2]. However the details of the IVR enhancements remain sketchy [1].

Described here are new data for positron annihilation resolved as a function of positron energy. The initial motivation was to understand the annihilation spectra in the small molecules $\mathrm{CCl}_{4}$ and $\mathrm{CBr}_{4}$ that are known to have very large annihilation rates for thermal positrons at $293 \mathrm{~K}$. For these targets, simple theoretical estimates for VFRs and IVR-enhanced VFRs appeared to be incapable of explaining the magnitudes of the observed rates. In particular, the positron binding energy in $\mathrm{CBr}_{4}$ is expected to be greater than all of the mode energies. This makes the VFR energies in Eq. (1) negative, thus shutting off the resonant process.

Based upon the study described here, we are led to conclude that broad quasicontinuous spectra of multimode vibrations (e.g., combinations and overtones) are responsible for the observed behavior. Further, this mechanism appears to be universal in the sense that it is present, at least at a significant level, in the spectra of most molecules studied to date. Qualitative agreement is obtained between the experimental data and the predictions of a model that assumes positron capture into (and possible detachment from) a statistically complete ensemble of strongly mixed multiquantum vibrational states. We term this mechanism statistical multimode resonant annihilation (SMRA). The more general implications of these results are discussed.

The experimental procedures are described in [1]. An energy tunable, trap-based positron beam is used that has a total energy spread $\sim 40 \mathrm{meV}$ (FWHM). Target gases are studied in a gas cell viewed by a detector that records singlequantum annihilation events. The measured test-gas pressure and the path length as viewed by the detector are used to determine absolute annihilation rates. Systematic errors in these rates are estimated to be $\leq 15 \%$. Error bars shown are the expected $1 \sigma$ variations due to counting statistics. The test-gas temperature is $293 \mathrm{~K}$.

Annihilation rates are usually expressed as the dimensionless quantity $Z_{\text {eff }}$, which is the measured rate $\lambda$ normalized to the Dirac rate for a free electron gas of density equal to the number density $n$ of molecular gas [1]: $Z_{\text {eff }}=$ $\lambda\left(\pi r_{0}^{2} c n\right)^{-1}$, where $r_{0}$ is the classical electron radius, and $c$ is the speed of light. For a simple collision in which all $Z$ molecular electrons contribute at the Dirac rate, $Z_{\text {eff }}=Z$. It is well known that this crude estimate fails more often than not due to a variety of effects, and so $Z_{\text {eff }}$ should be regarded solely as a normalized rate.

Shown in Fig. 1 are measured annihilation rates $Z_{\text {eff }}$ as a function of positron energy $\varepsilon$, using a beam with energy resolution as described above, for chloroform $\left(\mathrm{CHCl}_{3}\right)$, carbon tetrachloride $\left(\mathrm{CCl}_{4}\right)$, bromoform $\left(\mathrm{CHBr}_{3}\right)$, and carbon tetrabromide $\left(\mathrm{CBr}_{4}\right)$. The key observation is that all four spectra have broad spectral components that rise 


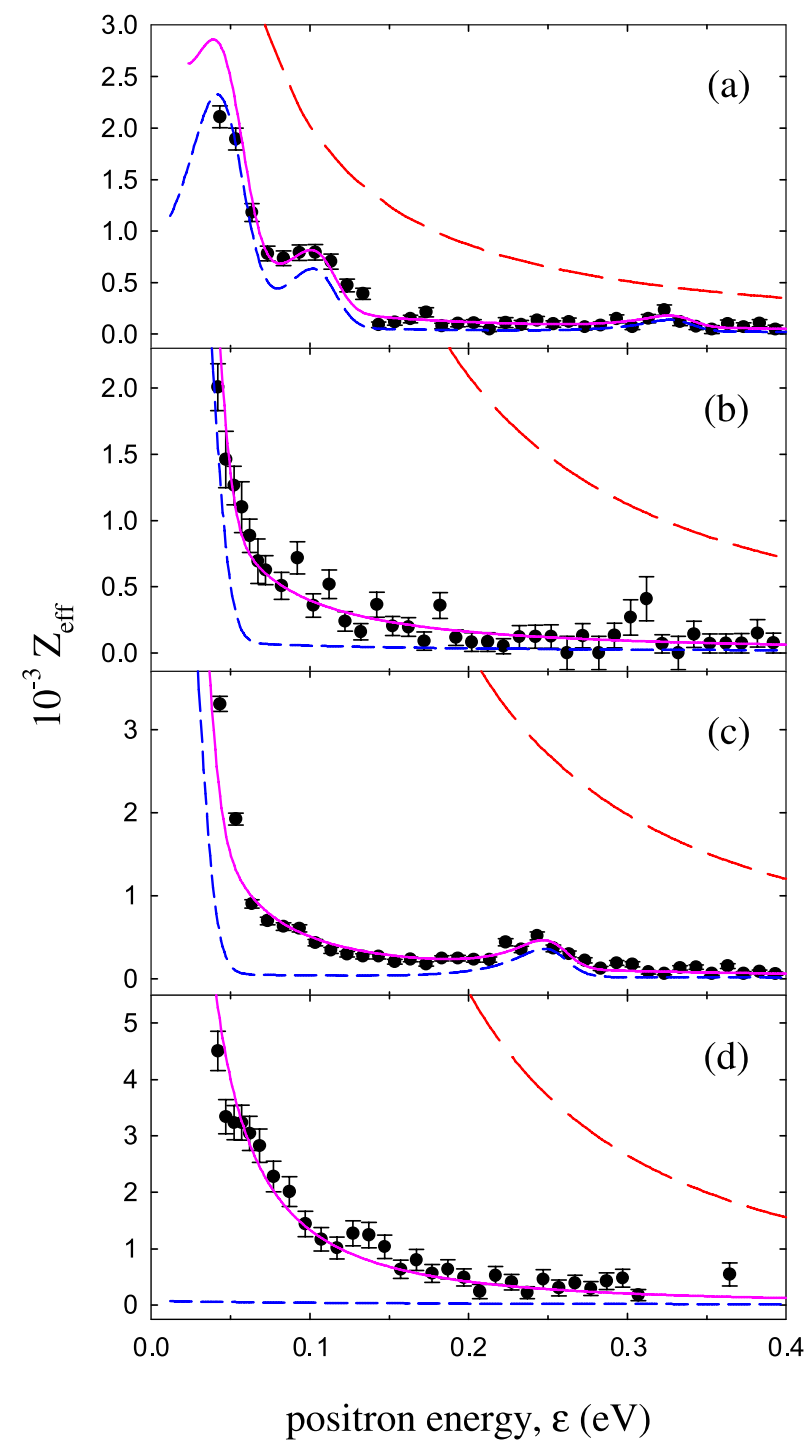

FIG. 1 (color online). (O) Measured annihilation rates $Z_{\text {eff }}$ as a function of incident positron energy for (a) chloroform $\mathrm{CHCl}_{3}$, (b) carbon tetrachloride $\mathrm{CCl}_{4}$, (c) bromoform $\mathrm{CHBr}_{3}$, and (d) carbon tetrabromide $\mathrm{CBr}_{4}$; (blue short-dashed lines) fundamental VFR and direct annihilation, Eq. (2); (red long-dashed lines) predictions of Eq. (3) for statistically complete multimode VFR; and (solid lines) combination of fundamental VFR and SMRA as discussed in the text. Binding energies are taken to be $50,75,130$, and $125 \mathrm{meV}$ for (a)-(d), respectively.

rapidly at lower energies. Here the measured $Z_{\text {eff }}$ values far exceed the number of molecular electrons (e.g., $Z=74$ for $\mathrm{CCl}_{4}$ and 146 for $\mathrm{CBr}_{4}$ ). The observed rates are much closer to those measured previously with thermal positrons at $293 \mathrm{~K}$, namely $Z_{\text {eff }} \sim 9,500$ and 40000 for $\mathrm{CCl}_{4}$ and $\mathrm{CBr}_{4}$, respectively [3].

A theory has been developed and tested that describes resonant annihilation due to single-mode VFRs [2,4]. For small polyatomic molecules in which the positron couples to all fundamental vibrations at a rate exceeding the annihilation rate, it gives $Z_{\text {eff }}(\varepsilon)$ as a sum of the resonant and direct annihilation contributions (in atomic units)

$$
\begin{aligned}
Z_{\mathrm{eff}}(\varepsilon) & =Z_{\mathrm{eff}}^{(\mathrm{res})}(\varepsilon)+Z_{\mathrm{eff}}^{(\mathrm{dir})}(\varepsilon) \\
& =\pi F \sum_{\nu} \sqrt{\frac{\varepsilon_{b}}{\varepsilon_{\nu}}} f\left(\varepsilon_{\nu}-\varepsilon\right)+\frac{F}{2\left(\varepsilon+\varepsilon_{b}\right)},
\end{aligned}
$$

where the sum is over all modes (including degeneracies), $f(\varepsilon)$ is the positron energy distribution, and $F \sim 0.7$ is a positron-electron overlap factor [1,2]. The second term in Eq. (2) estimates the direct "in-flight" annihilation of the scattered positron with the molecular electrons [1]. This broad nonresonant contribution is usually relatively small compared with that of VFR; for example, $Z_{\mathrm{eff}}^{(\mathrm{dir})}<200$ for beam energies $\varepsilon \geq 50 \mathrm{meV}$.

Shown in Fig. 1 by short-dashed lines are the predictions of Eq. (2), with the contribution of each mode adjusted for best fit, as discussed below. The values of $\varepsilon_{b}$ in $\mathrm{CHCl}_{3}$ and $\mathrm{CHBr}_{3}$ are determined from the downshifts of the VFR peaks in their measured $Z_{\text {eff }}$ spectra relative to the energies of the modes, as described by Eq. (1), giving $\varepsilon_{b}=50$ and $130 \mathrm{meV}$, respectively. The binding energies for $\mathrm{CCl}_{4}$ and $\mathrm{CBr}_{4}$, are estimated to be 75 and $125 \mathrm{meV}$. These values are based on the binding energies of $\mathrm{CHCl}_{3}$ and $\mathrm{CHBr}_{3}$ and an empirical formula for $\varepsilon_{b}$ developed previously (see Eq. (5) in Ref. [5]).

Comparisons of the data with the predictions of Eq. (2) indicate that there is appreciable spectral weight in $Z_{\text {eff }}(\varepsilon)$ in addition to the VFR. While the difference is modest for $\mathrm{CHCl}_{3}$, it is sizeable for $\mathrm{CCl}_{4}$ and $\mathrm{CHBr}_{3}$, and dominates for $\mathrm{CBr}_{4}$, where the predictions of Eq. (2) are barely visible on the scale of the data. Adding IVR enhancements of adjustable strengths to $Z_{\text {eff }}^{(\text {res }}$ (not shown) does little to improve the agreement. It is also difficult to see how this disagreement could be due to some kind of enhancement of $Z_{\mathrm{eff}}^{\text {(dir) }}$.

As can be seen from Fig. 1, the missing spectral weight appears to be broad in energy and rises at low energies. The SMRA model developed by Gribakin and Lee describes just such a contribution [6]. It assumes that the positron attaches to all energetically allowed multimode vibrational excitations (i.e., not just low-order combinations and overtones, as considered previously $[1,4,7]$ ). It is also assumed that all modes and combinations with sufficient energy to detach the positron can act subsequently as inelastic escape channels. Finally, it was assumed that the positron couples to all vibrational excitations with the same strength. This assumption is justified if the multimode excited states are strongly mixed due to anharmonicity, akin to the basic assumption for IVR [8].

The result for the SMRA contribution to $Z_{\text {eff }}$ is $[1,6]$

$$
Z_{\mathrm{eff}}^{\text {(stat) }}(\varepsilon)=\pi F \sqrt{\frac{\varepsilon_{b}}{\varepsilon}} \frac{\rho\left(\varepsilon+E_{v}+\varepsilon_{b}\right)}{N\left(\varepsilon+E_{v}\right)},
$$


where $E_{v}$ is the thermal energy in the target molecule, $\rho\left(\varepsilon+\varepsilon_{b}+E_{v}\right)$ is the energy density of entrance-channel multimode states populated at positron energy $\varepsilon$, and

$$
N\left(\varepsilon+E_{v}\right)=\int_{0}^{\varepsilon+E_{v}} \rho\left(E^{\prime}\right) d E^{\prime}
$$

is the total number of open inelastic escape channels. In the statistically complete limit, $\rho$ and $N$ can be calculated directly from the vibrational mode spectrum [6].

As an example, shown in Fig. 2(a) is the density $\rho(E)$ calculated for chloroform, where $E$ is the total vibrational energy of the positron-molecule complex. Both $\rho$ and $N$ grow rapidly as a function of energy. As a result, the ratio $\rho\left(\varepsilon+E_{v}+\varepsilon_{b}\right) / N\left(\varepsilon+E_{v}\right)$ in Eq. (3) increases sharply with increasing $\varepsilon_{b}$. This causes a fast rise of $Z_{\text {eff }}^{\text {(stat) }}$ with increasing molecular size, as this is accompanied by a steady increase in the binding energies (e.g., as observed in alkanes [1]).

Shown in Fig. 1 are the predictions of Eq. (3) for the SMRA contributions to the spectra for room-temperature target molecules convolved with the beam energy distribution. While the shapes of the missing broad components are similar to those given by the theory, the magnitudes of the latter are, in fact, too large. Thus, the solid lines in Fig. 1 show the sum of the VFR contributions of Eq. (2) and SMRA contributions of Eq. (3), adjusted for best fit. Except for the bend mode in bromoform, all VFRs have been reduced by factors ranging from 0.25 to 0.7 (cf., Fig. 1, short-dashed line), possibly indicating the presence

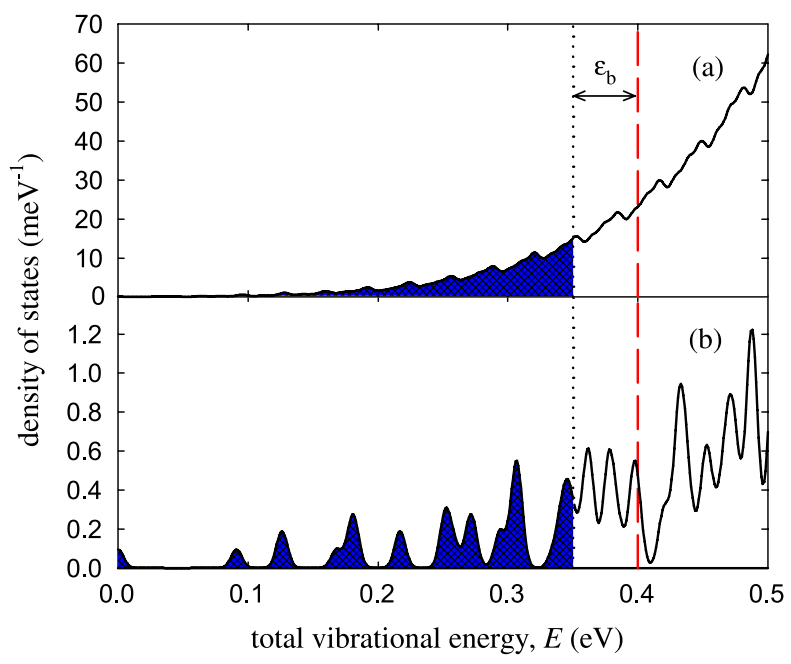

FIG. 2 (color online). Total density of vibrational states (smoothed by $10 \mathrm{meV}$ ) as a function of molecular vibrational energy $E$ for (a) chloroform $\left(\mathrm{CHCl}_{3}\right)$ and (b) methyl chloride $\left(\mathrm{CH}_{3} \mathrm{Cl}\right)$. Vertical dashed and dotted lines correspond to the total vibrational energy $E=\varepsilon+E_{v}+\varepsilon_{b}=350 \mathrm{meV}$ and maximum inelastic escape energy $\varepsilon+E_{v}=300 \mathrm{meV}$ for an assumed $\varepsilon_{b}=50 \mathrm{meV}$ for both molecules. The shaded area represents the total number of inelastic escape channels $N$. of inelastic escape channels [1]. The SMRA contributions have been reduced by factors $\eta=0.09,0.07,0.04,0.07$ for best fit in (a)-(d), respectively. The resulting predictions are in good agreement with the data.

The SMRA theory assumes that all multimode vibrations couple with equal strengths to the positron continuum and all such states can act as inelastic escape channels with the same coupling strengths [6]. The fact that $\eta<1$ suggests that some class of multimode vibrations has a substantially smaller excitation rate. This is quite possible in small polyatomics in which the vibrational spectrum density is not very high and the mode mixing and IVR are incomplete. If so, the fact that the spectral shapes appear to agree reasonably well with the data would indicate that this variation in coupling occurs relatively uniformly throughout the spectrum with no strong dependence on incident positron energy.

A noticeable effect, even in small molecules, is the absence of any underlying structure due to individual modes in $\rho$ and $N$, and hence in $Z_{\text {eff }}^{\text {(stat) }}$. This effect is illustrated in Fig. 2(b) for methyl chloride, chosen because of its spread-out mode structure due to high-energy $\mathrm{C}-\mathrm{H}$ modes. In this case, structure in $\rho$ is evident. However, even here, since $N$ is the integral over $\rho$ [cf. Eq. (4)], structure in $N$ will be less evident.

The effect of mode structure on the SMRA contribution is investigated further in Fig. 3, which shows $Z_{\text {eff }}^{\text {(stat) }}(\varepsilon)$ for $\mathrm{CHCl}_{3}$ and $\mathrm{CH}_{3} \mathrm{Cl}$ for two fictitious binding energies, $\varepsilon_{b}=20$ and $200 \mathrm{meV}$, spanning generously the range around the actual value, $\varepsilon_{b}=50 \mathrm{meV}$. In $\mathrm{CHCl}_{3}$ no structure is observed even at $\varepsilon_{b}=20 \mathrm{meV}$. While $\mathrm{CH}_{3} \mathrm{Cl}$ exhibits relatively prominent structure for $\varepsilon_{b}=20 \mathrm{meV}$,

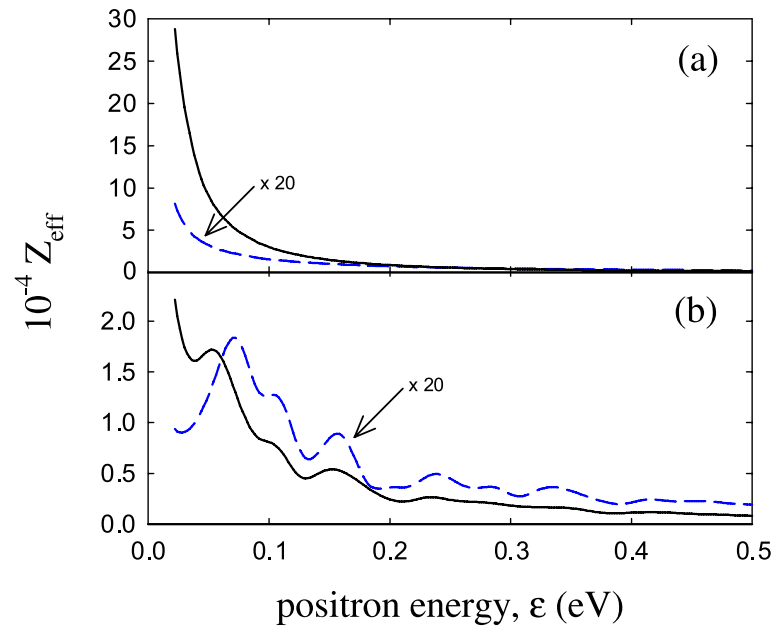

FIG. 3 (color online). SMRA contribution $Z_{\text {eff }}^{\text {(stat) }}$, Eq. (3), convolved with the experimental beam distribution, for (a) chloroform $\left(\mathrm{CHCl}_{3}\right)$ and (b) methyl chloride $\left(\mathrm{CH}_{3} \mathrm{Cl}\right)$, when artificially changing the binding energy from $20 \mathrm{meV}$ (dashed line) to $200 \mathrm{meV}$ (solid line). 


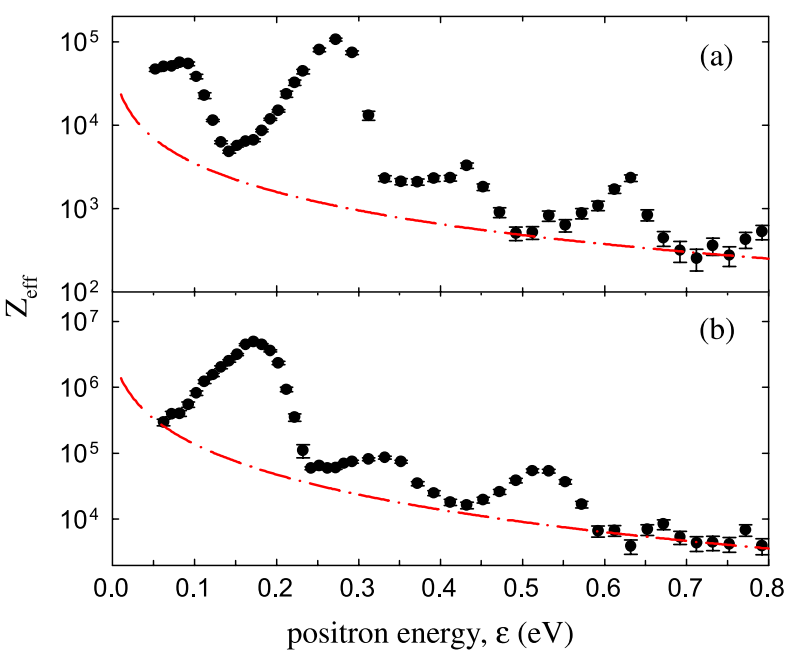

FIG. 4 (color online). (O) $Z_{\text {eff }}$ spectra for (a) hexane and (b) decane compared with (dash-dotted lines) the predictions of the SMRA model ( $\varepsilon_{b}=80 \mathrm{meV}$ and $170 \mathrm{meV}$, respectively), Eq. (3), scaled by $\eta=0.25$ for both molecules. The highest $Z_{\text {eff }}$ peak is due to the $\mathrm{C}-\mathrm{H}$ stretch modes, while the two smaller peaks at higher energies appear to be combination VFRs.

increasing the binding to $200 \mathrm{meV}$ leads to a spectrum with markedly less structure. This smoothing effect obscures the underlying mode spectrum in the SMRA contributions for all four molecules shown in Fig. 1, due to their relatively large binding energies and dominance of low-energy modes.

There is evidence that SMRA is present in the annihilation spectra of molecules over a wide range of species and sizes. The theory was originally formulated to explain annihilation spectra of alkanes, $\mathrm{C}_{n} \mathrm{H}_{2 n+2}$ [6]. However, in regions of IVR-enhanced single-mode VFRs, the $Z_{\text {eff }}$ in alkanes are larger in magnitude than the SMRA background by factors of 10 to 50. Nevertheless, careful inspection indicates that SMRA appears to be present at significant levels elsewhere in the spectra.

This is illustrated in Fig. 4 where data for hexane $\left(\mathrm{C}_{6} \mathrm{H}_{14}\right)$ and decane $\left(\mathrm{C}_{10} \mathrm{H}_{22}\right)$ are shown over a wider range of incident positron energies than covered by previous measurements. Note the appreciable $Z_{\text {eff }}$ values out to positron energies $\varepsilon \sim 0.8 \mathrm{eV}$. Also shown are the predictions of the SMRA model for $\eta=0.25$. The magnitude of this contribution explains well the spectral weight away from the fundamental VFR. The fact that $\eta$ is smaller than unity and differs significantly between the chemical species studied in Figs. 1 and 4 is a key issue and is not presently understood.

Note that the magnitude of the SMRA component increases by a factor of 30 going from hexane to decane. This increase with the number of carbons is primarily due to the increase in binding energy with molecular size rather than an increase in the number of modes and total vibrational density.
The measurements and analysis presented in Fig. 4 reveal structures in the resonantly enhanced $Z_{\text {eff }}$ at energies above the highest $(\mathrm{C}-\mathrm{H}$ mode) peak. While the peaks at energies $\epsilon \leq 0.3 \mathrm{eV}$ can be fit to the fundamental VFRs [1], those at higher energies appear to be due to positron capture by two-quantum or three-quantum overtones or combinations, which serve as extra doorways into the statistical multimode quasicontinuum.

Many molecules exhibit large enhancements of singlemode VFRs. This is thought to be due to IVR into a bath of "dark states" (i.e., vibrational states which do not couple directly, or couple weakly, to the positron continuum) [1]. It is possible that the multimode resonances of the SMRA model are these "dark states." If correct, this would represent an important step in developing a more complete model of the role of IVR in resonant annihilation on molecules. Another situation of interest is annihilation in molecules where $\varepsilon_{b}$ is sufficiently large so as to prohibit single-mode VFRs (e.g., in hydrocarbons where $\varepsilon_{b}$ is greater than the energy of the $\mathrm{C}-\mathrm{H}$ stretch mode). Such is the case for $\mathrm{CBr}_{4}$ in Fig. 1(d) as well as large polycyclic aromatic molecules relevant to annihilation in the interstellar medium $[9,10]$. In this case, SMRA should be the dominant contribution to the annihilation spectrum.

To summarize, evidence is presented here that statistical ensembles of multimode vibrations play an important role in resonant positron annihilation on molecules. The magnitude of the positron-molecule binding energy is a key parameter in determining the magnitude of their contribution. This dependence appears to be the reason that SMRA is prominent in the annihilation spectra of the halomethane and large alkane molecules discussed here.

Given the successes of the SMRA picture, there are several open questions, one of which is the degree to which the observed spectra reflect a statistically complete set of modes. Another, likely related, is the origin of the required attenuation factor $\eta$. Besides other approximations made in deriving Eq. (3), this factor may arise from the incomplete mixing of the multimode vibrations in small-tomoderate sized polyatomic molecules.

We thank E. A. Jerzewski for expert technical assistance. The research at UCSD is supported by the U. S. NSF, Grant No. PHY 10-68023.

[1] G. F. Gribakin, J. A. Young, and C. M. Surko, Rev. Mod. Phys. 82, 2557 (2010).

[2] G. F. Gribakin and C.M.R. Lee, Phys. Rev. Lett. 97, 193201 (2006).

[3] K. Iwata, R. G. Greaves, T. J. Murphy, M. D. Tinkle, and C. M. Surko, Phys. Rev. A 51, 473 (1995).

[4] J.A. Young, G.F. Gribakin, C.M. R. Lee, and C.M. Surko, Phys. Rev. A 77, 060702 (2008). 
[5] J. R. Danielson, J. A. Young, and C. M. Surko, J. Phys. B 42, 235203 (2009).

[6] G. F. Gribakin and C. M. R. Lee, Eur. Phys. J. D 51, 51 (2008).

[7] S. d'A. Sanchez, M. A.P. Lima, and M. T.d. N. Varella, Phys. Rev. Lett. 107, 103201 (2011).
[8] D. J. Nesbitt and R. W. Field, J. Phys. Chem. 100, 12735 (1996).

[9] K. Iwata, R. G. Greaves, and C. M. Surko, Can. J. Phys. 74, 407 (1996).

[10] N. Guessoum, P. Jean, and W. Gillard, Mon. Not. R. Astron. Soc. 402, 1171 (2010). 